\title{
Edge-maximality of power graphs of finite cyclic groups
}

\author{
Brian Curtin • G.R. Pourgholi
}

Received: 18 September 2012 / Accepted: 27 November 2013 / Published online: 10 December 2013

(C) Springer Science+Business Media New York 2013

\begin{abstract}
We show that among all finite groups of any given order, the cyclic group of that order has the maximum number of edges in its power graph.
\end{abstract}

Keywords Cyclic group $\cdot p$-Group $\cdot$ Greatest prime divisor $\cdot$ Least prime divisor

\section{Introduction}

In this paper, we resolve in the affirmative a conjecture of Mirzargar et al. [13, Conjecture 2] concerning the number of edges in the power graph of a finite group. Motivated by the work of Kelarev and Quinn [9-12], Chakrabarty, Ghosh, and Sen [8] introduced undirected power graphs to study semigroups and groups. Other relevant work includes $[6,7]$. The reader is encouraged to see [1] which surveys the literature to date.

Definition 1.1 Let $G$ be a finite group. Let $\langle g\rangle$ denote the cyclic subgroup of $G$ generated by $g \in G$.

\footnotetext{
B. Curtin

Department of Mathematics and Statistics, University of South Florida, Tampa, FL 33620, USA e-mail: bcurtin@usf.edu

G.R. Pourgholi ( ()

School of Mathematics, Statistics and Computer Science, University of Tehran,

Tehran 14155-6455, Iran

e-mail: pourgholi@ut.ac.ir

G.R. Pourgholi

e-mail: pourgholi@alumni.ut.ac.ir

G.R. Pourgholi

e-mail: gh.reza.pourgholi@gmail.com
} 
(i) The directed power graph $\overrightarrow{\mathcal{P}}(G)$ of $G$ is the directed graph with vertex set $G$ and directed edge set $\vec{E}(G)=\{(g, h) \mid g, h \in G, h \in\langle g\rangle-\{g\}\}$. That is, there is an edge from one group element to a second whenever the second is a positive power of the first and distinct from the first.

(ii) The undirected power graph (or power graph) $\mathcal{P}(G)$ of $G$ is the undirected graph with vertex set $G$ and edge set $E(G)=\{\{g, h\} \mid(g, h) \in \vec{E}(G)$ or $(h, g) \in$ $\vec{E}(G)\}$. That is, two distinct group elements are adjacent whenever one of them is a positive power of the other.

We recall the following property of directed power graphs of cyclic groups.

Theorem 1.2 [2, Main Theorem] Among all finite groups of a given order, the cyclic group of that order has the maximum number of edges in its directed power graph.

Our main theorem, which resolves [13, Conjecture 2], is the undirected analog of Theorem 1.2.

Theorem 1.3 Among all finite groups of a given order, the cyclic group of that order has the maximum number of edges in its power graph.

Theorem 1.3 gives that (ii) implies (iii) in the following. The others are trivial.

Corollary 1.4 Let $G$ be a finite group. Then the following are equivalent for all $n \geq 1$ :

(i) $\mathcal{P}(G) \cong \mathcal{P}\left(\mathbb{Z}_{n}\right)$.

(ii) $|E(G)|=\left|E\left(\mathbb{Z}_{n}\right)\right|$.

(iii) $G \cong \mathbb{Z}_{n}$.

One special case of Theorem 1.3 is already known.

Theorem 1.5 [8, Theorem 2.12] A finite group has a complete power graph if and only if it is cyclic and has prime power order.

\section{Edges in power graphs}

Let $G$ be a finite group. For $g \in G$, let o $(g)$ denote the order of $g$ as a group element and let $\operatorname{deg}(g)$ denote the degree of $g$ as a vertex of $\mathcal{P}(G)$. Throughout $\phi(n)$ shall denote the Euler totient function of the natural number $n$.

Pick $g \in G$. Observe that $g$ has out-degree $o(g)-1$ since there is a directed edge from $g \in G$ to each element of $\langle g\rangle-\{g\}$. There is a directed edge from each $h \in$ $G-\{g\}$ to $g$ for which $g \in\langle h\rangle$, so the in-degree of $g$ is $|\{h \in G-\{g\} \mid g \in\langle h\rangle\}|$. To account for directed edges which give the same undirected edge in the power graph of $G$, we introduce the following set. 
Definition 2.1 The set of bidirectional edges $\overleftrightarrow{E}(G)$ of $\overrightarrow{\mathcal{P}}(G)$ is set of unordered pairs $\{\{g, h\} \mid(g, h) \in \vec{E}(G)$ and $(h, g) \in \vec{E}(G)\}$. That is, $\overleftrightarrow{E}(G)$ consists of pairs of distinct elements, each of which is a positive power of the other.

Lemma 2.2 Let $G$ be a finite group, and let $g, h$ be distinct elements of $G$. Then $\{g, h\} \in \overleftrightarrow{E}(G)$ if and only if $\langle g\rangle=\langle h\rangle$

Proof Straightforward from the definition of adjacency in the directed power graph.

Lemma 2.3 [8, Theorem 4.2] Let $G$ be a finite group of order $n$. Then

$$
\begin{aligned}
|\vec{E}(G)| & =\sum_{g \in G}(\mathrm{o}(g)-1), \\
|\overleftrightarrow{E}(G)| & =\frac{1}{2} \sum_{g \in G}(\phi(\mathrm{o}(g))-1), \\
|E(G)| & =|\vec{E}(G)|-|\overleftrightarrow{E}(G)|=\frac{1}{2} \sum_{g \in G}(2 \mathrm{o}(g)-\phi(\mathrm{o}(g))-1) .
\end{aligned}
$$

Proof The sum in (1) adds out-degrees of vertices, and thus counts each directed edge once. Now (2) follows from Lemma 2.2 and the fact that a cyclic group of order $\mathrm{o}(g)$ has $(\phi(\mathrm{o}(g)))$-many generators. Indeed, $\phi(\mathrm{o}(g))-1$ such edges leave $g$, and summing over all $G$ double counts these edges. For the first equality in (3), count the edges in the directed power graph, and subtract one for each pair of oppositely oriented directed edges to avoid double counting. The second equality in (3) follows from (1) and (2).

We give the number of edges in the undirected power graph of $\mathbb{Z}_{n}$. We use the following notation.

Notation 2.4 Let $n$ be a positive integer. Write $n=p_{1}^{\alpha_{1}} p_{2}^{\alpha_{2}} \cdots p_{k}^{\alpha_{k}}$ for primes $p_{1}<$ $p_{2}<\cdots<p_{k}$. Let $q=p_{1}$ and $p=p_{k}$ be the least and greatest prime divisors of $n$, and abbreviate $\beta=\alpha_{1}$ and $\alpha=\alpha_{k}$.

It is well-known (see [3, p. 27], for instance) that

$$
\phi(n)=p_{1}^{\alpha_{1}-1}\left(p_{1}-1\right) p_{2}^{\alpha_{2}-1}\left(p_{2}-1\right) \cdots p_{k}^{\alpha_{k}-1}\left(p_{k}-1\right) .
$$

As a consequence, we have [3, p. 28]

$$
\phi(n m)=\phi(n) \phi(m) \frac{\operatorname{gcd}(n, m)}{\phi(\operatorname{gcd}(n, m))} .
$$


Lemma 2.5 (See also [5], p. 143, Exercise 5) With Notation 2.4,

$$
\begin{gathered}
\sum_{z \in \mathbb{Z}_{n}} \mathrm{o}(z)=\sum_{d \mid n} \phi(d) d=\prod_{h=1}^{k} \frac{p_{h}^{2 \alpha_{h}+1}+1}{p_{h}+1}, \\
\sum_{z \in \mathbb{Z}_{n}} \phi(\mathrm{o}(z))=\sum_{d \mid n} \phi(d)^{2}=\prod_{h=1}^{k} \frac{p_{h}^{2 \alpha_{h}}\left(p_{h}-1\right)+2}{p_{h}+1} .
\end{gathered}
$$

Proof For each $z \in \mathbb{Z}_{n}, \mathrm{o}(z)$ is a divisor $d$ of $n$. For each divisor $d$, there are $\phi(d)$ many other elements of $\mathbb{Z}_{n}$ with the same order. Thus the first equality in (6) holds. Similarly, for each of the $\phi(d)$-many elements of $\mathbb{Z}_{n}$ with the same order as $z$, $\phi(\mathrm{o}(z))=\phi(d)$. Thus the first equality in (7) holds.

When $n=1$, its only divisor is 1 , and $\phi(1)=\phi(1)^{2}=1$. Thus both sums on the left are 1 . There are no prime divisors of 1 , so the product on the right is empty, and hence 1 . Thus both second equalities holds when $k=0$. Assume that $n$ has $k \geq 1$ distinct prime divisors and that both second equalities holds for all $n$ with at most $k-1$ distinct prime divisors.

Partition the divisors of $n$ according to the highest power of $q$ which divides it. As $d$ runs over $d \mid n$ with $q^{\ell} \mid f$ and $q^{\ell+1} \nmid f$, we have $d=f q^{\ell}$ as $f$ runs over the divisors of $n / q^{\beta}$. Since $q \nmid n / q^{\beta}$, (4) gives that $\phi\left(f q^{\ell}\right)=q^{\ell-1}(q-1) \phi(f)$. Compute

$$
\begin{aligned}
\sum_{d \mid n} \phi(d) d & =\sum_{\ell=0}^{\beta} \sum_{f \mid n / q^{\beta}} \phi\left(q^{\ell} f\right) f q^{\ell}=\sum_{\ell=0}^{\beta} \phi\left(q^{\ell}\right) q^{\ell} \sum_{f \mid n / q^{\beta}} \phi(f) f \\
& =\left(1+\sum_{\ell=1}^{\beta} q^{\ell-1}(q-1) q^{\ell}\right) \sum_{f \mid n / q^{\beta}} \phi(f) f,
\end{aligned}
$$

and

$$
\begin{aligned}
\sum_{\ell=1}^{\beta} q^{2 \ell-1}(q-1) & =\sum_{\ell=0}^{\beta-1}(q-1) q^{2 \ell+1}=\frac{q-1}{q} \cdot \sum_{\ell=0}^{\beta-1}\left(q^{2}\right)^{\ell}=q(q-1) \cdot \frac{\left(q^{2 \beta}-1\right)}{q^{2}-1} \\
& =q \cdot \frac{q^{2 \beta}-1}{q+1}=\frac{q^{2 \beta+1}+1}{q+1}-1 .
\end{aligned}
$$

Now the second equality in (6) follows by induction.

Similarly,

$$
\begin{aligned}
\sum_{d \mid n} \phi(d)^{2} & =\sum_{\ell=0}^{\beta} \sum_{f \mid n / q^{\beta}} \phi\left(q^{\ell} f\right)^{2}=\sum_{\ell=0}^{\beta} \phi\left(q^{\ell}\right)^{2} \sum_{f \mid n / q^{\beta}} \phi(f)^{2} \\
& =\left(1+\sum_{\ell=1}^{\beta}\left(q^{\ell-1}(q-1)\right)^{2}\right) \sum_{f \mid n / q^{\beta}} \phi(f)^{2},
\end{aligned}
$$


and

$$
\sum_{\ell=1}^{\beta}\left(q^{\ell-1}(q-1)\right)^{2}=(q-1)^{2} \sum_{\ell=0}^{\beta-1} q^{2 \ell}=(q-1)^{2} \frac{q^{2 \beta}-1}{q^{2}-1}=\frac{(q-1)\left(q^{2 \beta}-1\right)}{q+1} .
$$

Now the second equality in (7) follows by induction.

Corollary 2.6 With Notation 2.4,

$$
\begin{aligned}
\left|E\left(\mathbb{Z}_{n}\right)\right| & =\sum_{d \mid n} \phi(d)\left(d-\frac{\phi(d)}{2}\right)-\frac{n}{2} \\
& =\prod_{h=1}^{k} \frac{p_{h}^{2 \alpha_{h}+1}+1}{p_{h}+1}-\frac{1}{2} \prod_{h=1}^{k} \frac{p_{h}^{2 \alpha_{h}}\left(p_{h}-1\right)+2}{p_{h}+1}-\frac{n}{2} .
\end{aligned}
$$

Lemma 2.7 With Notation 2.4, pick $z \in \mathbb{Z}_{n}$, and write $e=\mathrm{o}(z)$. Then

$$
\begin{aligned}
\operatorname{deg}(z) & =e-1-\phi(e)+\sum_{d \mid n / e} \phi(d e) \\
& =e-\phi(e)-1+\phi(e) \sum_{d \mid n / e} \phi(d) \frac{\operatorname{gcd}(d, e)}{\phi(\operatorname{gcd}(d, e))} .
\end{aligned}
$$

Proof The term o $(z)-1=e-1$ is the out-degree of $z$. There is a directed edge from each element $x \in \mathbb{Z}_{n}$ to $z$ whenever $\mathrm{o}(z) \mid \mathrm{o}(x)$. For such an $x, \mathrm{o}(x)$ is $\mathrm{o}(z)$ times a divisor of $n / \mathrm{o}(z)$. There are $\phi(k \mathrm{o}(z))$-many generators of $\langle x\rangle$. Thus the in-degree of $z$ is $\sum_{d \mid n / e} \phi(d e)$. However, to avoid double counting when dropping the orientation, we must exclude those elements with the same order as $z$, i.e., the case $k=1$ where $\phi(k e)=\phi(e)$. Hence the first equality holds. The second equality follows from (5) since the summand for $d=1$ is 1 .

Lemma 2.8 Let $G$ be a group, and let $H$ be a subgroup of $G$.

(i) $\overrightarrow{\mathcal{P}}(H)$ is an induced subgraph of $\overrightarrow{\mathcal{P}}(G)$.

(ii) All out-edges from an element of $H$ terminate at an element of $H$.

(iii) $\mathcal{P}(H)$ is an induced subgraph of $\mathcal{P}(G)$.

In particular, the adjacencies and non-adjacencies between elements of $H$ are the same in $\overrightarrow{\mathcal{P}}(H)$ and $\overrightarrow{\mathcal{P}}(G)$, and similarly for $\mathcal{P}(H)$ and $\mathcal{P}(G)$.

Proof Straightforward from the definitions.

\section{Some inequalities}

We develop some inequalities. 
Lemma 3.1 Let $G$ be a finite group. Then the following hold.

(i) $|G| \leq|E(G)|+1$ with equality if and only if $G$ is an elementary Abelian 2group.

(ii) $|E(G)| \leq|\vec{E}(G)|$ with equality if and only if $G$ is an elementary Abelian 2group.

(iii) $2|\overleftrightarrow{E}(G)| \leq|\vec{E}(G)|-|G|+1$ with equality if and only if $G$ is an elementary Abelian 2-group or $G \cong \mathbb{Z}_{p}$ for some prime $p$.

Proof (i) Every nonidentity element of $G$ is adjacent to the identity, so $|G| \leq$ $|E(G)|+1$. Equality holds if and only if every nonidentity element of $G$ has order two.

(ii) Every undirected edge arises from a directed edge, so the inequality holds. By (3), $|E(G)|=|\vec{E}(G)|$ if and only if there are no bidirectional edges if and only if for all $g \in G, g$ is the only generator of $\langle g\rangle$ if and only if for all $g \in G$, one is the only number both less than and coprime to $o(g)$ if and only if every element of $G$ has order two.

(iii) The $(|G|-1)$-many edges to the identity are not bidirectional, and the bidirectional edges come from pairs of directed edges. Thus $2|\overleftrightarrow{E}(G)| \leq|\vec{E}(G)|-|G|+1$. If some $g \in G$ has composite order, say o $(g)=p m$ for a prime $p$ and $m>1$, then the edge between $g$ and $g^{p}$ is not bidirectional. Now suppose that $|\overleftrightarrow{E}(G)| \neq 0$. Then equality holds in (iii) if and only if every edge not incident to the identity is bidirectional if and only if every nonidentity element generates $G$ if and only if $G \cong \mathbb{Z}_{p}$ for some prime $p>2$. Now $|\overleftrightarrow{E}(G)|=0$ if and only if $G$ has no element of order greater than 2 if and only if $G$ is an elementary Abelian 2-group.

The remaining inequalities in this section pertain to $\mathbb{Z}_{n}$, and so are number theoretic in nature.

Lemma 3.2 [2, Main Theorem] Let $G$ be a group of finite order $n$. Then $\sum_{g \in G} \mathrm{o}(g) \leq$ $\sum_{z \in \mathbb{Z}_{n}} \mathrm{O}(z)$, with equality if and only if $G$ is isomorphic to $\mathbb{Z}_{n}$.

Theorem 1.2 is a straightforward consequence of (1) and Lemma 3.2. Our next goal is to improve the bound $\sum_{d \mid n} \phi(d) d>n^{2} / p$ given in [2, Lemma D].

Corollary 3.3 With Notation 2.4, assume that $n \neq 2^{\alpha}$ with $\alpha>1$. Then

$$
\sum_{d \mid n} \phi(d) d \geq\left(\prod_{h=1}^{k} \frac{p_{h}+1}{p_{h}}\right) \cdot \frac{n^{2}}{p} \geq\left(\frac{q+1}{q}\right) \cdot \frac{n^{2}}{p} .
$$

Proof We induct on the number $k$ of distinct prime divisors of $n$. If $n=p^{\alpha}$ for $p>2$, then (9) holds since the summand $\phi\left(p^{\alpha}\right) p^{\alpha}=p^{2 \alpha-1}(p-1)$ alone is larger than $(p+1 / p)\left(p^{2 \alpha} / p\right)$. If $n=2$, then (9) is easily verified. By assumption $n \neq 2^{\alpha}$ for $\alpha>1$, since otherwise (9) fails. This establishes the initial step for the induction on $k$. 
Suppose that $k \geq 2$, so $n / q^{\beta}$ is not a power of two. Also suppose that

$$
\sum_{d \mid n / q^{\beta}} \phi(d) d=\prod_{h=2}^{k}\left(\frac{p_{h}^{2 \alpha_{h}+1}+1}{p_{h}+1}\right) \geq\left(\prod_{h=2}^{k} \frac{p_{h}+1}{p_{h}}\right) \cdot \frac{\left(n / q^{\beta}\right)^{2}}{p} .
$$

Now

$$
\begin{aligned}
\sum_{d \mid n} \phi(d) d & =\prod_{h=1}^{k}\left(\frac{p_{h}^{2 \alpha_{h}+1}+1}{p_{h}+1}\right)=\left(\frac{q^{2 \beta+1}+1}{q+1}\right) \cdot \prod_{h=2}^{k}\left(\frac{p_{h}^{2 \alpha_{h}+1}+1}{p_{h}+1}\right) \\
& \geq\left(\frac{q^{2 \beta+1}+1}{q+1}\right) \cdot\left(\prod_{h=2}^{k} \frac{p_{h}+1}{p_{h}}\right) \cdot\left(\frac{n^{2}}{q^{2 \beta} p}\right) \\
& =\left(\frac{q^{2 \beta+1}+1}{q^{2 \beta+1}+q^{2 \beta}}\right)\left(\prod_{h=2}^{k} \frac{p_{h}+1}{p_{h}}\right) \frac{n^{2}}{p} \\
& \geq \frac{q+1}{q} \cdot\left(\prod_{h=2}^{k} \frac{p_{h}+1}{p_{h}}\right) \cdot \frac{n^{2}}{p}=\left(\prod_{h=1}^{k} \frac{p_{h}+1}{p_{h}}\right) \cdot \frac{n^{2}}{p} .
\end{aligned}
$$

The first inequality follows by induction, and the second holds since $\left(p_{h}+1\right) / p_{h}=$ $1+1 / p_{h}>1$.

Lemma 3.4 With Notation 2.4, pick $z \in \mathbb{Z}_{n}$, and write $e=\mathrm{o}(z)$. Then

$$
\operatorname{deg}(z) \geq e-1+\phi(e)\left(\frac{n}{e}-1\right)
$$

with equality if and only if $e$ and $n / e$ are coprime.

Proof Consider (8). The term $\operatorname{gcd}(d, e) / \phi(\operatorname{gcd}(d, e))$ is at least 1 with equality if and only if $\operatorname{gcd}(d, e)=1$. If $n / e$ has any divisors not coprime to $e$, then the inequality must be strict.

Lemma 3.5 With Notation 2.4,

$$
\phi(n) \geq \frac{n}{p},
$$

with equality if and only if $n=2^{\alpha} 3^{\beta}$ with $\alpha \geq 1$ and $\beta \geq 0$.

Proof If $n=p^{\alpha}$, then $\phi\left(p^{\alpha}\right)=p^{\alpha-1}(p-1) \geq p^{\alpha-1}=p^{\alpha} / p$. The inequality holds in this case, with equality if and only if $p=2$. Now assume that $n$ has $k \geq 2$ distinct prime factors. By $(4), \phi(n) / n=\left(p_{1}-1\right)\left(p_{2}-1\right) \cdots\left(p_{k}-1\right) /\left(p_{1} p_{2} \cdots p_{k}\right)$. Note that $p_{i+1}-1 \geq p_{i-1}(1 \leq i \leq k-1)$, with equality precisely when $k=2$, $p_{1}=2$, and $p_{2}=3$. Telescoping the middle terms gives $\phi(n) / n \geq\left(p_{1}-1\right) / p_{k}=$ $(q-1) / p \geq 1 / p$, with equality under the stated conditions, i.e., $n=2^{\alpha} 3^{\beta}$ for positive $\alpha$ and nonnegative $\beta$. 
Lemma 3.6 With Notation 2.4, assume that $n$ is not equal to $2^{\alpha}$ with $\alpha>1$. Then

$$
\begin{aligned}
& \sum_{z \in \mathbb{Z}_{n}} \operatorname{deg}(z) \geq 2 \frac{n^{2}}{p}+\frac{n}{p}-1 \quad \text { if } \phi(n) \leq n / q, \\
& \sum_{z \in \mathbb{Z}_{n}} \operatorname{deg}(z)>(n-1)\left(\frac{n}{q}+1\right) \quad \text { if } \phi(n)>n / q .
\end{aligned}
$$

Proof For any prime $p$ (other than 2), both $\phi\left(p^{\alpha}\right)=p^{\alpha-1}(p-1)>p^{\alpha} / p$ by (4) and $\left|E\left(\mathbb{Z}_{p^{\alpha}}\right)\right|=p^{\alpha}\left(p^{\alpha}-1\right) / 2>p^{\alpha} / p$ by Theorem 1.5 . Thus we may assume that $n$ is not a prime power.

Each of the $\phi(n)$-many generators of $\mathbb{Z}_{n}$ has degree $n-1$, as does the identity. So summing over the generators and the identity gives

$$
\sum_{z} \operatorname{deg}(z)=\phi(n)(n-1)+n-1=(\phi(n)+1)(n-1) .
$$

If $\phi(n)>n / q$, then

$$
\sum_{z \in \mathbb{Z}_{n}} \operatorname{deg}(z)>(n-1)\left(\frac{n}{q}+1\right)>\frac{n^{2}}{q}
$$

By (8), $z \in \mathbb{Z}_{n}$ has degree $\operatorname{deg}(z)=\mathrm{o}(z)-1-\phi(\mathrm{o}(z))+\sum_{d \mid n / \mathrm{o}(z)} \phi(d \mathrm{o}(z))$. For each of the $(n-\phi(n)-1)$-many nonidentity nongenerators $z$ of $\mathbb{Z}_{n}$, the summand corresponding to $d=1$ is $\phi(\mathrm{o}(z))$ and to $d=n / \mathrm{o}(z)$ is $\phi(n)$. By $(10), \phi(n) \geq n / p$. Thus $\operatorname{deg}(z) \geq \mathrm{o}(z)-1+n / p$. Equality holds for all nonidentity nongenerators if and only if $n=6$. Now

$$
\begin{aligned}
\sum_{z \in \mathbb{Z}_{n}} \operatorname{deg}(z) & \geq\left(\sum_{z \in \mathbb{Z}_{n}} \mathrm{o}(z)\right)-n+(n-\varphi(n)-1) \frac{n}{p}+n-1 \\
& \geq\left(\sum_{z \in \mathbb{Z}_{n}} \mathrm{o}(z)\right)+\frac{n^{2}}{p}-(\phi(n)-1) \frac{n}{p}-1 .
\end{aligned}
$$

If $\phi(n) \leq n / q$, then, by Corollary 3.3,

$$
\sum_{z \in \mathbb{Z}_{n}} \operatorname{deg}(z) \geq \frac{q+1}{q} \frac{n^{2}}{p}+\frac{n^{2}}{p}-\left(\frac{n}{q}-1\right) \frac{n}{p}-1=2 \frac{n^{2}}{p}+\frac{n}{p}-1 .
$$

Recall that for any undirected graph $\Gamma$

$$
|E(\Gamma)|=\frac{1}{2} \sum_{g \in \Gamma} \operatorname{deg}(g) .
$$


Lemma 3.7 With Notation 2.4, suppose $\phi(n)>n / q$. Then $n$ is odd.

Proof In light of (4), $\phi(n)>n / q$ if and only if $\left(p_{1}-1\right) \cdots\left(p_{k}-1\right)>p_{2} \cdots p_{k}$. Assume $q=p_{1}=2$, so $p_{1}-1=1$. Then the above inequality gives $\left(p_{2}-1\right) \cdots$ $\left(p_{k}-1\right)>p_{2} \cdots p_{k}$, which is impossible. Therefore $n$ is odd.

Lemma 3.8 Assume that $r$ and $s$ are natural numbers such that $s \mid r$. Then $2 r-$ $\phi(r)-1 \geq 2 s-\phi(s)-1$, with equality if and only if $s=r$.

Proof Write $r=s t$, and observe that $\phi(s t) \leq \phi(s) t$. Then

$$
2 r-\phi(r)-1 \geq 2 s t-\phi(s) t-1=t(2 s-\phi(s))-1 \geq 2 s-\phi(s)-1 .
$$

Observe that if $t>1$, then this inequality is strict.

\section{Direct products}

Let $G$ be a finite group. Suppose $G=U \times V$ is the direct product of normal subgroups $U$ and $V$. We view the underlying set of $G$ as the Cartesian product $U \times V=\{u v \mid u \in U, v \in V\}$, where we use juxtaposition (and suggestive notation) to avoid confusion with directed edges in a power graph. We use - for the group product on $U \times V$, namely $u v \cdot u^{\prime} v^{\prime}=\left(u u^{\prime}\right)\left(v v^{\prime}\right)$. We write $g \rightarrow h$ when $(g, h) \in \vec{E}(G)$, that is, when $h=g^{a}$ but $h \neq g$ for some $a$.

Lemma 4.1 Let $G$ be a finite group, and let $U$ and $V$ be subgroups of $G$. Define

$$
\begin{aligned}
D= & \left\{\left(u v, u^{\prime} v^{\prime}\right) \mid u u^{\prime} \in \vec{E}(U), v v^{\prime} \in \vec{E}(V)\right\} \\
& \cup\left\{\left(u v, u^{\prime} v\right) \mid u u^{\prime} \in \vec{E}(U), v \in V\right\} \\
& \cup\left\{\left(u v, u v^{\prime}\right) \mid u \in U, v v^{\prime} \in \vec{E}(V)\right\}, \\
B= & \left\{\left\{u v, u^{\prime} v^{\prime}\right\} \mid u u^{\prime} \in \overleftrightarrow{E}(U), v v^{\prime} \in \overleftrightarrow{E}(V)\right\} \\
& \cup\left\{\left\{u v, u^{\prime} v\right\} \mid u u^{\prime} \in \overleftrightarrow{E}(U), v \in V\right\} \\
& \cup\left\{\left\{u v, u v^{\prime}\right\} \mid u \in U, v v^{\prime} \in \overleftrightarrow{E}(V)\right\} .
\end{aligned}
$$

Suppose $G$ is the direct product $U \times V$ of $U$ and $V$. Then

$$
\begin{gathered}
\vec{E}(U \times V) \subseteq D, \\
\overleftrightarrow{E}(U \times V) \subseteq B .
\end{gathered}
$$

Proof Let $u, u^{\prime} \in U$ and $v, v^{\prime} \in V$. First suppose $u v \rightarrow u^{\prime} v^{\prime}$ in $\overrightarrow{\mathcal{P}}(U \times V)$, so for some positive integer $c, u^{\prime} v^{\prime}=(u v)^{\cdot c}=u^{c} v^{c}$. The product is direct, so $u^{\prime}=u^{c}$ and $v^{\prime}=v^{c}$. Thus one of the following holds: (1d) $u \rightarrow u^{\prime}$ in $\overrightarrow{\mathcal{P}}(U)$ and $v \rightarrow v^{\prime}$ in $\overrightarrow{\mathcal{P}}(V)$, 
(2d) $u^{\prime}=u$ and $v \rightarrow v^{\prime}$ in $\overrightarrow{\mathcal{P}}(V),(3 \mathrm{~d}) u \rightarrow u^{\prime}$ in $\overrightarrow{\mathcal{P}}(U)$ and $v^{\prime}=v$, or (4d) $u^{\prime}=u$ and $v^{\prime}=v$. Thus (11) holds. Now suppose $u^{\prime} v^{\prime} \leftrightarrow u v$ in $\overleftrightarrow{\mathcal{P}}(U \times V)$. As above, one of the following holds: (1u) $u^{\prime} \leftrightarrow u$ in $\overleftrightarrow{\mathcal{P}}(U)$ and $v^{\prime} \leftrightarrow v$ in $\overleftrightarrow{\mathcal{P}}(V)$, (2u) $u^{\prime}=u$ and $v^{\prime} \leftrightarrow v$ in $\overleftrightarrow{\mathcal{P}}(V),(3 \mathrm{u}) u^{\prime} \leftrightarrow u$ in $\overleftrightarrow{\mathcal{P}}(U)$ and $v^{\prime}=v$, or $(4 \mathrm{u}) u^{\prime}=u$ and $v^{\prime}=v$ Thus (12) holds.

Lemma 4.2 With reference to Lemma 4.1, suppose $G=U \times V$. Then the following hold.

(i) $\vec{E}(U \times V)=D$ if and only if $(|U|,|V|)=1$.

(ii) If $(|U|,|V|)=1$, then $\overleftrightarrow{E}(U \times V)=B$.

Proof (i) Suppose that $\operatorname{gcd}(|U|,|V|)=1$. Let $u \rightarrow u^{\prime}$ in $\overrightarrow{\mathcal{P}}(U)$ and $v \rightarrow v^{\prime}$ in $\overrightarrow{\mathcal{P}}(V)$. Then $u v \rightarrow u^{\prime} v^{\prime}$ in $\overrightarrow{\mathcal{P}}(U \times V)$. Also for each $u \in U$ and $v \in V$ we have $u v \rightarrow u v^{\prime}$ in $\overrightarrow{\mathcal{P}}(U \times V)$ and $u v \rightarrow u^{\prime} v$ in $\overrightarrow{\mathcal{P}}(U \times V)$. Therefore $D \subseteq \vec{E}(U \times V)$, and thus equality holds in this case.

We now show that there are no other instances of equality. Suppose some prime $p$ divides $\operatorname{gcd}(|U|,|V|)$. Then $U$ and $V$ contain respective elements $u$ and $v$ of order $p$. Observe that there is no edge $u v \rightarrow u e$ although it appears in the third subset in the definition of $D$. Thus the inclusion is strict unless no prime divides $\operatorname{gcd}(|U|,|V|)$, i.e., $\operatorname{gcd}(|U|,|V|)=1$.

(ii) The proof is similar to the first part of the proof of (i).

Corollary 4.3 With reference to Lemma 4.1, suppose that $G=U \times V$ and $(|U|,|V|)=1$. Then

(i) The edge set of the directed power graph of $U \times V$ has size

$$
|\vec{E}(U \times V)|=|\vec{E}(U)| \cdot|\vec{E}(V)|+|\vec{E}(U)| \cdot|V|+|U| \cdot|\vec{E}(V)| .
$$

(ii) The set of bidirectional edges of the directed power graph of $U \times V$ has size

$$
|\overleftrightarrow{E}(U \times V)|=2|\overleftrightarrow{E}(U)| \cdot|\overleftrightarrow{E}(V)|+|\overleftrightarrow{E}(U)| \cdot|V|+|U| \cdot|\overleftrightarrow{E}(V)|
$$

Proof Referring to the proof of (11), there are $|\vec{E}(U)| \cdot|\vec{E}(V)|$ pairs in case (1d), $|U| \cdot|\vec{E}(V)|$ pairs in case $(2 \mathrm{~d}),|\vec{E}(U)| \cdot|V|$ pairs in case $(3 \mathrm{~d})$, but no possible edges in case (4d). Therefore, (i) holds. Referring to the proof of (12), there are $2|\overleftrightarrow{E}(U)|$. $|\overleftrightarrow{E}(V)|$ pairs in case (1u) since for all $\left\{u, u^{\prime}\right\} \in \overleftrightarrow{\mathcal{P}}(U)$ and $\left\{v, v^{\prime}\right\} \in \overleftrightarrow{\mathcal{P}}(V)$ we have that $u^{\prime} v^{\prime} \leftrightarrow u v$ and $u^{\prime} v \leftrightarrow u v^{\prime}$ are distinct bidirectional edges in $\overleftrightarrow{E}(G)$. There are $|U| \cdot|\overleftrightarrow{E}(V)|$ pairs in case $(2 \mathrm{u})$, and $|\overleftrightarrow{E}(U)| \cdot|V|$ pairs in case $(3 \mathrm{u})$, but no possible edges in case $(4 \mathrm{u})$. Thus (ii) holds.

Lemma 4.4 Let $U, V$, and $V^{\prime}$ be finite groups with $|V|=\left|V^{\prime}\right|$ and $(|U|,|V|)=1$. Then

$$
\begin{aligned}
|E(U \times V)|-\left|E\left(U \times V^{\prime}\right)\right|= & (|\vec{E}(U)|-2|\overleftrightarrow{E}(U)|)\left(|\vec{E}(V)|-\left|\vec{E}\left(V^{\prime}\right)\right|\right) \\
& +(2|\overleftrightarrow{E}(U)|+|U|)\left(|E(V)|-\left|E\left(V^{\prime}\right)\right|\right) .
\end{aligned}
$$


Proof Expand $|E(U \times V)|-\left|E\left(U \times V^{\prime}\right)\right|$ with (3):

$$
\begin{aligned}
|E(U \times V)|-\left|E\left(U \times V^{\prime}\right)\right|= & (|\vec{E}(U \times V)|-|\vec{E}(U \times V)|) \\
& -\left(|\overleftrightarrow{E}(U \times V)|-\left|\overleftrightarrow{E}\left(U \times V^{\prime}\right)\right|\right) .
\end{aligned}
$$

Since $|V|=\left|V^{\prime}\right|$, Corollary 4.3 gives

$$
\begin{aligned}
& |\vec{E}(U \times V)|-\left|\vec{E}\left(U \times V^{\prime}\right)\right|=(|\vec{E}(U)|+|U|)\left(|\vec{E}(V)|-\left|\vec{E}\left(V^{\prime}\right)\right|\right), \\
& |\overleftrightarrow{E}(U \times V)|-\left|\overleftrightarrow{E}\left(U \times V^{\prime}\right)\right|=(2|\overleftrightarrow{E}(U)|+|U|)\left(|\overleftrightarrow{E}(V)|-\left|\overleftrightarrow{E}\left(V^{\prime}\right)\right|\right)
\end{aligned}
$$

In the second line, use (3) to write $|\overleftrightarrow{E}(V)|=|\vec{E}(V)|-|E(V)|$, and similarly for $\left|\overleftrightarrow{E}\left(V^{\prime}\right)\right|$. Thus

$$
|\overleftrightarrow{E}(U \times V)|-\left|\overleftrightarrow{E}\left(U \times V^{\prime}\right)\right|=(2|\overleftrightarrow{E}(U)|+|U|)\left(|\overleftrightarrow{E}(V)|-\left|\overleftrightarrow{E}\left(V^{\prime}\right)\right|\right)
$$

Combining the above and simplifying gives the desired result.

Corollary 4.5 With reference to Lemma 4.4, suppose $|E(V)| \geq\left|E\left(V^{\prime}\right)\right|$ and $|\vec{E}(V)| \geq\left|\vec{E}\left(V^{\prime}\right)\right|$. Then $|E(U \times V)| \geq\left|E\left(U \times V^{\prime}\right)\right|$, with equality if and only if $|E(V)|=\left|E\left(V^{\prime}\right)\right|$ and $|\vec{E}(V)|=\left|\vec{E}\left(V^{\prime}\right)\right|$.

Proof Observe that $|\vec{E}(U)|>2|\overleftrightarrow{E}(U)|$ since each bidirectional edge is counted twice in $\vec{E}(U)$ and since $\vec{E}(U)$ contains one-way edges from each element of $U$ to the identity. Also $\vec{E}(U)$, includes the one-way edges from each element of $U$ to the identity. Clearly, $2|\overleftrightarrow{E}(U)|+|U|>0$. With the given assumptions, Lemma 4.4 gives that $|E(U \times V)|-\left|E\left(U \times V^{\prime}\right)\right| \geq 0$, so the result follows. Equality holds if and only if $|E(V)|=\left|E\left(V^{\prime}\right)\right|$ and $|\vec{E}(V)|=\left|\vec{E}\left(V^{\prime}\right)\right|$ since all terms on the right are positive, so the right side is zero if and only if these equalities hold.

\section{Semidirect products}

We recall semidirect products. Recall that a group $G$ is the (internal) semidirect product of a normal subgroup $U$ and a subgroup $V$ if and only if $G=U V$ and $U \cap V=\{e\}$ [14]. To uniquely determine $G$ from $U$ and $V$, we specify a homomorphism $\varphi: V \rightarrow \operatorname{Aut}(U)$. As is the custom, write $G=U \rtimes_{\varphi} V$ in this situation. When there is no ambiguity, we write $\varphi v$ for $\varphi(v)$ and place such automorphisms as a superscript of the element of $U$ to which it is applied.

The elements of $G=U \rtimes_{\varphi} V$ can be identified with the Cartesian product of the underlying sets of $U$ and $V$, just as is the case for the direct product. We use $*$ for the group product on $U \rtimes_{\varphi} V$, namely $u v * u^{\prime} v^{\prime}=\left(u\left(u^{\prime}\right)^{\varphi v}\right)\left(v v^{\prime}\right)$. We write $(u v)^{\cdot c}$ and $(u v)^{* c}$ to denote the $c$ th powers of $u v$ under the corresponding operations. We write o. $(u v)$ and $\mathrm{o}_{*}(u v)$ to denote the order of $u v$ relative to the corresponding 
multiplication, and we write $(u v)^{-1}$ and $(u v)^{*-1}$ for the corresponding inverses. With this notation,

$$
\begin{aligned}
u v^{* c} & =\left(u u^{\varphi v} u^{\varphi v^{2}} \cdots u^{\varphi v^{c-2}} u^{\varphi v^{c-1}}\right)\left(v^{c}\right), \\
(u v)^{*-1} & =\left(u^{-1}\right)^{\varphi v^{-1}}\left(v^{-1}\right) .
\end{aligned}
$$

Lemma 5.1 Suppose that $G$ is a finite group and that $G=U \rtimes_{\varphi} V$ is the semidirect product of a normal cyclic subgroup $U$ and a subgroup $V$. Pick $u \in U$ and $v \in V$. Then $u^{\varphi v}=u^{r}$ for some $r$, and $(u v)^{* c}=u^{t} v^{c}$, where $t=1+r+r^{2}+\cdots+r^{c-1}$.

Proof Consider the subgroup $\langle u\rangle$ of $U$. Since $U$ is cyclic and since $\langle u\rangle$ is the unique subgroup of $U$ of its order, it must be the case that $\langle u\rangle$ is a characteristic subgroup of $U$. Thus $u^{\varphi v}=u^{r}$ for some $r$. Now $u^{\varphi v^{b}}=u^{r^{b}}$. With this we compute

$$
\begin{aligned}
(u v)^{* c} & =\left(u u^{\varphi v} u^{\varphi v^{2}} \cdots u^{\varphi v^{c-2}} u^{\varphi v^{c-1}}\right)=\left(u^{1} u^{r} u^{r^{2}} \cdots u^{r^{c-1}}\right)\left(v^{c}\right) \\
& =\left(u^{1+r+r^{2}+\cdots+r^{c-1}}\right)\left(v^{c}\right)=u^{t} v^{c} .
\end{aligned}
$$

Lemma 5.2 Suppose that $G$ is a finite group and that $G=U \rtimes_{\varphi} V$ is the semidirect product of a normal cyclic subgroup $U$ and a subgroup $V$. Then

$$
\begin{aligned}
& \vec{E}\left(U \rtimes_{\varphi} V\right) \subseteq D, \\
& \overleftrightarrow{E}\left(U \rtimes_{\varphi} V\right) \subseteq B .
\end{aligned}
$$

In particular, if $(|U|,|V|)=1$, then $E\left(U \rtimes_{\varphi} V\right) \subseteq E(U \times V)$ and $\overleftrightarrow{E}\left(\mathbb{Z}_{|U|} \rtimes_{\varphi}\right.$ $\left.\mathbb{Z}_{|V|}\right) \subseteq \overleftrightarrow{E}\left(\mathbb{Z}_{|U|} \times \mathbb{Z}_{|V|}\right)$

Proof Pick $u, u^{\prime} \in U$ and $v, v^{\prime} \in V$, and say $u^{\varphi v}=u^{r}$. Suppose $u v \rightarrow u^{\prime} v^{\prime}$ in $\overrightarrow{\mathcal{P}}\left(U \rtimes_{\varphi} V\right)$. Then $u^{\prime} v^{\prime}=(u v)^{* c}=u^{t} v^{c}$ for some $t$ as in Lemma 5.1. Since the product is semidirect, $u^{\prime}=u^{t}$ and $v^{\prime}=v^{c}$. Arguing as in the proof of Lemma 4.1, we reach the same conclusion.

Lemma 5.3 Suppose that $G$ is a finite group and that $G=U \rtimes_{\varphi} V$ is the semidirect product of a normal Abelian subgroup $U$ and a subgroup $V$. Assume $U$ and $V$ have coprime orders. Then for all $u \in U$ and $v \in V, \mathrm{o}_{*}(u v) \mid \mathrm{o} .(u v)$.

Proof To prove the result we show that $(u v)^{* 0 .(u v)}$ is the identity of $U \rtimes_{\varphi} V$. Note that $\mathrm{o} .(u v)=\mathrm{o}(u) \mathrm{o}(v) / \operatorname{gcd}(\mathrm{o}(u), \mathrm{o}(v))=\mathrm{o}(u) \mathrm{o}(v)$. By (13),

$$
\begin{aligned}
(u v)^{* \mathrm{o}(v)} & =\left(u u^{\varphi v} u^{\varphi v^{2}} \cdots u^{\varphi v^{\mathrm{o}(v)-2}} u^{\varphi v^{\mathrm{o}(v)-1}}\right)\left(v^{\mathrm{o}(v)}\right) \\
& =\left(u u^{\varphi v} u^{\varphi v^{2}} \cdots u^{\varphi v^{\mathrm{o}(v)-2}} u^{\varphi v^{\mathrm{o}(v)-1}}\right)(e) .
\end{aligned}
$$


Thus

$$
\begin{aligned}
(u v)^{* \mathrm{o} .(u v)} & =\left[\left(u u^{\varphi v} u^{\varphi v^{2}} \cdots u^{\varphi v^{\mathrm{o}(v)-2}} u^{\varphi v^{\mathrm{o}(v)-1}}\right)(e)\right]^{* \mathrm{o}(u)} \\
& =\left(u^{\mathrm{o}(u)}\left(u^{\varphi v}\right)^{\mathrm{o}(u)}\left(u^{\varphi v^{2}}\right)^{\mathrm{o}(u)} \cdots\left(u^{\varphi v^{\mathrm{o}(v)-2}}\right)^{\mathrm{o}(u)}\left(u^{\varphi v^{\mathrm{o}(v)-1}}\right)^{\mathrm{o}(u)}\right)(e) \\
& =(e)(e),
\end{aligned}
$$

since $U$ is Abelian, as required.

For later use we recall a couple facts involving semidirect products.

Lemma 5.4 [14, Theorem 10.30] (The Schur-Zassenhaus theorem) Let $G$ be a finite group, and let $K$ be a normal subgroup of $G$ with $(|K|,|G: K|)=1$. Then $G$ is a semidirect product of $K$ and $G / K$. In particular, there exists a subgroup $H$ of $G$ with order $|G: K|$ satisfying $G=H K$ and $H \cap K=\{e\}$.

Lemma 5.5 [4, Theorem 1.2(i)] Let $p$ be an odd prime, and suppose that $P$ is a non-Abelian p-group with a cyclic subgroup of index $p$. Then $P \cong \mathbb{Z}_{p^{\alpha-1}} \rtimes_{\varphi} \mathbb{Z}_{p}$, the center of $P$ has order $|Z(P)|=p^{\alpha-2}$, and $P$ has presentation

$$
P \cong M_{p^{\alpha}}=\left\langle a, b \mid a^{p^{\alpha-1}}=b^{p}=1, b^{-1} a b=a^{1+p^{\alpha-2}}\right\rangle .
$$

\section{Some group theory}

We need a few more results from group theory.

Definition 6.1 Let $p$ be a prime. Let $G$ be a finite group, and let $P$ be a Sylow $p$ subgroup of $G$. A $p$-complement in $G$ is a subgroup with index equal to the order of a Sylow $p$-subgroup.

Theorem 6.2 [14, Theorem 10.21] (Burnside's transfer theorem) With the notation of Definition 6.1, if $P \subseteq Z\left(N_{G}(P)\right)$, then $G$ has a normal p-complement.

Corollary 6.3 [14, Corollary 10.24] With the notation of Definition 6.1, suppose $G$ contains a cyclic $q$-Sylow subgroup, where $q$ is the least prime divisor of $|G|$. Then $G$ has a normal q-complement.

Lemma 6.4 A finite group containing a cyclic subgroup of prime index has a normal Sylow subgroup.

Proof Let $G$ be a finite group and suppose $C$ is a cyclic subgroup of prime index $p$ in $G$. Induct on the number of distinct prime factors of $|G|$. If $G$ is a $p$-group, then $G$ itself is a normal Sylow $p$-subgroup. Assume that there is a prime $r$ different from $p$ which divides $|G|$.

Let $R$ be a Sylow $r$-subgroup of $C$. Now $|G: R|=p \cdot|C: R|$ is not divisible by $r$, so $R$ is a Sylow $r$-subgroup of $G$. We are done if $R$ is normal in $G$, so assume that 
this is not the case. Now $R$ is normal in the cyclic subgroup $C$, so $C \leq N_{G}(R)<G$. Since $C$ has prime index in $G$, it must be the case that $N_{G}(R)=C$. In particular, $R \leq Z\left(N_{G}(R)\right)$, so $G$ has a normal $r$-complement $N$ by Burnside's transfer theorem. Thus $R N=G$, and hence $C N=G$ as well.

Now $N \cap C$ is cyclic since $C$ is, and $|N: N \cap C|=|C N: C|=|G: C|=p$. Suppose $N \cap C$ is nontrivial. Then by induction $N$ has a nontrivial normal Sylow subgroup $S$. In fact, $S$ is characteristic in $N$, so $S$ is normal in $G$. Observe that $S$ is a Sylow subgroup of $G$ since $|G: N|$ and $|N|$ are coprime.

Suppose $N \cap C=1$. Then $|G|=|N C|=|N||C| /|N \cap C|=(|G| /|R|)(|G| / p) / 1$, so $|G|=p r^{\alpha}$ for some positive power $\alpha$. Now $R$ and $P$ are cyclic subgroups of $G$, so Corollary 6.3 implies that if $r<p$ then $G$ has a normal $r$-complement, and if $p<r$ then $G$ has a normal $p$-complement. In either case, there is a normal Sylow subgroup of $G$.

Lemma 6.5 Let $G$ be a finite group of order $n$. Suppose $n$ is not a power of 2 , and let $r>2$ be a prime divisor of $n$. Suppose that $G$ contains both a cyclic $r$-complement and a normal $r$-Sylow subgroup which itself contains a cyclic subgroup of index $r$. Then there is a bijection $\lambda: G \rightarrow \mathbb{Z}_{n}$ such that $\mathrm{o}(g) \mid \mathrm{o}(\lambda(g))$ for each $g \in G$.

Proof Let $U$ be a cyclic $r$-complement of $G$. Let $R$ denote a normal Sylow $r$ subgroup of $G$ which contains a cyclic subgroup $C$ with index $r$. Say $|R|=r^{\alpha}$. By consideration of group orders, $G=R U$ and $R \cap U=\{e\}$. Since $R$ is normal in $G$, both $G=R \rtimes_{\varphi} U$ and $G / R \cong U$.

Suppose $R$ is non-Abelian. By Lemma 5.5, the center $Z(R)$ of $R$ has order $r^{\alpha-2}$. Now $Z(R) \subseteq C$ since otherwise by consideration of indices $R=Z(R) C$; this would imply that $R$ is Abelian, contrary to our assumption. Hence $Z(R)$ is cyclic; moreover, it is the unique subgroup of $C$ with index $r$. Now $Z(R)$ is a characteristic subgroup of the normal (characteristic) subgroup $R$, so $Z(R)$ is normal in $G$. Since $Z(R)$ is a normal cyclic subgroup of $G$, Lemma 5.3 gives that $o_{*}(a b) \mid$ o. $(a b)$ for all $a b \in$ $Z(R) \rtimes_{\varphi} U$. Observe that $Z(R) \times U$ is cyclic. Thus we may take $\lambda$ restricted to $Z(R) \rtimes_{\varphi} U$ to be a bijection from $Z(R) \rtimes_{\varphi} U$ to $Z(R) \times U \cong \mathbb{Z}_{|Z(R)|} \times \mathbb{Z}_{|U|} \subseteq \mathbb{Z}_{n}$ for which $\mathrm{o}(x) \mid \mathrm{o}(\lambda(x))$ for each $x \in Z(R) \rtimes_{\varphi} U$.

Now since $G=R U$, we can write $G=Z(R) U \cup(R-Z(R)) U$. Suppose that $x \in(R-Z(R)) U$. Then $x=r u$ where $r \in R-Z(R)$ and $u \in U$. Since $R$ is not cyclic it contains no element of order $r^{\alpha}$. Hence the order of $x$ is divisible by $r^{\alpha-1} \mathrm{o}(u)$. We identify $\mathbb{Z}_{|U|}$ with $U$, so $\mathbb{Z}_{n}=\mathbb{Z}_{|Z(R)|} U \cup\left(\mathbb{Z}_{r^{\alpha}}-\mathbb{Z}_{|Z(R)|}\right) U$. Since the elements of $\mathbb{Z}_{r^{\alpha}}-\mathbb{Z}_{|Z(R)|}$ have order $r^{\alpha-1}$ or $r^{\alpha}$ it follows that $r^{\alpha-1} \mathrm{o}(u) \mid \mathrm{o}(z) \mathrm{o}(u)$ for each $z \in \mathbb{Z}_{r^{\alpha}}-\mathbb{Z}_{|Z(R)|}$. Thus any bijection from $G-\left(Z(R) \rtimes_{\varphi} U\right)$ to $\mathbb{Z}_{n}-\mathbb{Z}_{|Z(R)|} \mathbb{Z}_{|U|}$ will extend $\lambda$ to all of $G$ with the desired properties.

Now suppose $R$ is Abelian. If $R$ is cyclic then by Lemma 5.3 we have nothing to prove. Suppose $R$ is not cyclic. Then Lemma 5.3 gives that $o_{*}(a b) \mid o(a b)$ for all $a b \in R \rtimes_{\varphi} U$. Now it is enough to show that there is a bijection from $R \times U$ to $\mathbb{Z}_{n}$ with the desired property. By assumption, $R$ has a cyclic subgroup of index $r$. Thus $R \cong \mathbb{Z}_{r^{\alpha-1}} \times \mathbb{Z}_{r}$, and $R \times U \cong \mathbb{Z}_{r^{\alpha-1}} \times \mathbb{Z}_{r} \times U$. Now if we write $R \times U=$ $\left(R-\mathbb{Z}_{r^{\alpha-1}}\right) U \cup \mathbb{Z}_{r^{\alpha-1}} U$ then arguing as in the above we reach the same conclusion. 
Lemma 6.6 Let $G$ be a finite group, and suppose that there is a bijection $\lambda: G \rightarrow$ $\mathbb{Z}_{n}$ such that $\mathrm{o}(g) \mid \mathrm{o}(\lambda(g))$ for each $g \in G$. Then $|E(G)|=\left|E\left(\mathbb{Z}_{n}\right)\right|$ if and only if $G \cong \mathbb{Z}_{n}$.

Proof Suppose $|E(G)|=\left|E\left(\mathbb{Z}_{n}\right)\right|$. By Lemma 3.8, for all $g \in G, 2 \mathrm{o}(g)-\phi(\mathrm{o}(g))-$ $1 \leq 2 \mathrm{o}(\lambda(g))-\phi(\mathrm{o}(\lambda(g)))-1$. By (3), and since $\lambda$ is a bijection $|E(G)|=$ $\sum_{g \in G} 2 \mathrm{o}(g)-\phi(\mathrm{o}(g))-1=\sum_{g \in G} 2 \mathrm{o}(\lambda(g))-\phi(\mathrm{o}(\lambda(g)))-1=\left|E\left(\mathbb{Z}_{n}\right)\right|$. This equality and the preceding inequality imply that for all $g \in G, 2 \mathrm{o}(g)-\phi(\mathrm{o}(g))=$ $20(\lambda(g))-\phi(o(\lambda(g)))$.

Pick a generator $z$ of $\mathbb{Z}_{n}$, and let $g=\lambda^{-1}(z)$. Then $2 \mathrm{o}(g)-\phi(\mathrm{o}(g))=2 \mathrm{o}(\lambda(g))-$ $\phi(\mathrm{o}(\lambda(g)))=2 \mathrm{o}(z)-\phi(\mathrm{o}(z))=2 n-\phi(n)$. Suppose $G$ is not cyclic. Then $\mathrm{o}(g)<n$ and $\mathrm{o}(g)$ divides $n=\mathrm{o}(z)$. Lemma 3.8 implies that $2 \mathrm{o}(g)-\phi(\mathrm{o}(g))<2 n-\phi(n)$. This contradicts the above. Thus $G$ must be cyclic, and hence isomorphic to $\mathbb{Z}_{n}$. The converse is clear.

\section{Proof of main theorem}

The proof of Theorem 1.3 is developed in a series of technical lemmas.

Notation 7.1 Let $G$ be a finite group of order $n$, and adopt the conventions of Notation 2.4 for the prime factorization of $n$.

Lemma 7.2 With Notation 7.1, the following hold.

(i) No cyclic group is a counterexample to Theorem 1.3.

(ii) No group of prime power order is a counterexample to Theorem 1.3.

Proof Part (i) is clear, and Part (ii) follows from Theorem 1.5.

Lemma 7.3 With Notation 7.1, suppose $G=P \rtimes_{\phi} T$ is the semidirect product of a normal cyclic Sylow subgroup $P$ and a subgroup $T$ with order coprime to that of $P$. Then $G$ is not a minimal counterexample to Theorem 1.3.

Proof Suppose $G$ is a minimal counterexample to Theorem 1.3. By Lemma 5.2, $|E(P \times T)| \geq\left|E\left(P \rtimes_{\varphi} T\right)\right|=|E(G)| \geq\left|E\left(\mathbb{Z}_{n}\right)\right|$.

Note that $P$ is isomorphic to $\mathbb{Z}_{|P|}$. By construction $\operatorname{gcd}(|P|,|T|)=1$. For the sake of comparison, let $T^{\prime}=\mathbb{Z}_{|T|}$, and observe that $P \times T^{\prime}$ is isomorphic to $\mathbb{Z}_{n}$. Identify $\mathbb{Z}_{n}$ and $P \times T^{\prime}$.

Suppose $T$ is not cyclic. Since $|T|<|G|$, and since $G$ is assumed to be a minimal counterexample, $|E(T)|<\left|E\left(T^{\prime}\right)\right|$. Also, by Theorem 1.2, $|\vec{E}(T)|<\left|\vec{E}\left(T^{\prime}\right)\right|$. Now by Corollary 4.5, $|E(P \times T)|<\left|E\left(P \times T^{\prime}\right)\right|=\left|E\left(\mathbb{Z}_{n}\right)\right|$. This implies that $\left|E\left(\mathbb{Z}_{n}\right)\right|>$ $\left|E\left(\mathbb{Z}_{n}\right)\right|$, which is absurd. This contradiction leads to the conclusion that in this case, $G$ is not a minimal counterexample to Theorem 1.3.

Suppose $T$ is cyclic. Then $G$ is the semidirect product of cyclic subgroups of coprime orders. Note that $G$ is not cyclic by Lemma 7.2. Now is $P \times T$; in fact, $P \times T$ 
is isomorphic to $\mathbb{Z}_{n}$ since $\operatorname{gcd}(|P|,|T|)=1$. Thus $|E(G)| \leq\left|E\left(\mathbb{Z}_{n}\right)\right|=|E(P \times T)|$. It remains to show that equality does not hold.

Suppose $|E(G)|=\left|E\left(\mathbb{Z}_{n}\right)\right|$. Since $T$ is a cyclic $p$-complement and $P$ is a cyclic Sylow $p$-subgroup, Lemma 5.3 gives that there is a bijection $\theta: G \rightarrow \mathbb{Z}_{n}$ such that $\mathrm{o}(g) \mid \mathrm{o}(\theta(g))$ for all $g \in G$. Now Lemma 6.6, leads to the conclusion that $G$ is cyclic. This contradiction implies that in this case, $G$ is not a minimal counterexample to Theorem 1.3 either. The result follows.

Lemma 7.4 With Notation 7.1, if there exists $g \in G$ with $\mathrm{o}(g)>n / p$, then $G$ is not a minimal counterexample to Theorem 1.3.

Proof Since o $(g)>n / p$, we have $|G:\langle g\rangle|=|G| / \mathrm{o}(g)<p$. Thus $\langle g\rangle$ contains a Sylow $p$-subgroup $P$ of $G$. In particular, $P$ is cyclic and normalized by $\langle g\rangle$; hence, $P \triangleleft G$. By Lemma 5.4, $G=P \rtimes_{\varphi} T$ (semidirect product). The result follows from Lemma 7.3.

Lemma 7.5 With Notation 7.1, if $\phi(n) \leq \frac{n}{q}$, then $G$ is not a minimal counterexample to Theorem 1.3.

Proof Suppose $G$ is a minimal counterexample to Theorem 1.3. Then $|E(G)| \geq$ $\left|E\left(\mathbb{Z}_{n}\right)\right|$. Note that $G$ is not cyclic and $n$ is not a prime power by Lemma 7.2. In particular, $n$ is not a power of 2 .

By assumption and Lemmas 2.3 and 3.6,

$$
\begin{aligned}
\sum_{g \in G} 2 \mathrm{o}(g)-\phi(\mathrm{o}(g))-1 & =\sum_{g \in G} \operatorname{deg}(g)=2|E(G)| \geq 2\left|E\left(\mathbb{Z}_{n}\right)\right| \\
& =\sum_{z \in \mathbb{Z}_{n}} \operatorname{deg}(z) \geq 2 \frac{n^{2}}{p}+\frac{n}{p}-1 .
\end{aligned}
$$

Cancel -1 on the right and $-\phi(o(e))=-1$ on the left, and then drop the remaining $-\phi(\mathrm{o}(g))$ on the left. Add $n=\sum_{g \in G} 1$ to both sides to find

$$
\sum_{g \in G} 20(g)>2 \frac{n^{2}}{p}+\frac{n}{p}+n .
$$

Thus there is at least one $g \in G$ for which $\mathrm{o}(g)>n / p$. The result follows from Lemma 7.4.

Lemma 7.6 With Notation 7.1, if $\phi(n)>\frac{n}{q}$, then $G$ is not a minimal counterexample to Theorem 1.3.

Proof Suppose $G$ is a counterexample of minimal order. Then arguing as in Lemma 7.5, we find

$$
\sum_{g \in G} 2 o(g)-\phi(o(g))-1 \geq(n-1)\left(\frac{n}{q}+1\right) .
$$


Each term $\phi(o(g))$ is at least one, so we may add $2 n$ to both sides and then drop all remaining contributions of $-\phi(\mathrm{o}(g))$. Also subtract 2, the contribution of $g=e$, from each side:

$$
\sum_{g \in G-\{e\}} 20(g) \geq(n-1)\left(\frac{n}{q}+1\right)+2 n-2 .
$$

Thus among the $n-1$ terms on the left, there is at least one nonidentity $g$ with $\mathrm{o}(g) \geq n / 2 q+1 / 2+1$. In particular, $\mathrm{o}(g)>n / 2 q$.

Suppose $p>2 q$. Then $n / p<n / 2 q$, and thus $\mathrm{o}(g)>n / 2 q>n / p$. In this case, the result follows from Lemma 7.4. Note $p \neq 2 q$ since $p$ is prime.

Now suppose $p<2 q$ (this case occurs when $n$ is the product of twin primes, for example). Then $\mathrm{o}(g)>n / 2 q$, so $|G:\langle g\rangle|=|G| / \mathrm{o}(g)<n /(n / 2 q)=2 q$. Note that $n$ is odd by Lemma 3.7, and thus $|G:\langle g\rangle|$ is a prime number, say $s$. Now by Lemma 6.4, $G$ has a normal $r$-Sylow subgroup $R$ for some prime $r$. By Lemma 5.4, $G$ contains an $r$-complement $U$. Thus $G=R \rtimes_{\varphi} U$.

Note that $\langle g\rangle$ contains all Sylow $t$-subgroups of $G$ for $t \neq s$. If $r \neq s$, then $R \subseteq\langle g\rangle$, so it is cyclic. The hypotheses of Lemma 7.3 are satisfied by $R$ and $U$, so we conclude that $G$ is not a minimal counterexample in this case.

Suppose that $r=s$. Say $|R|=r^{\beta}$. Since $|G:\langle g\rangle|=r$, the subgroup $H=\left\langle g^{r^{\beta-1}}\right\rangle$ has order $|H|=|G| / r^{\beta}$. Thus $H$ is a cyclic $r$-complement. Note that $q>2$ by Lemma 3.7.

Now Lemma 6.5 gives a bijection, say again $\lambda$, from $G$ to $\mathbb{Z}_{n}$ such that o $(g) \mid$ $\mathrm{o}(\lambda(g))$ for all $g \in G$. In this case Lemma 6.6 leads to the conclusion that $G$ is cyclic. This contradiction implies that $G$ is not a minimal counterexample to Theorem 1.3.

We are now ready to prove Theorem 1.3.

Proof Since Lemmas 7.5 and 7.6 exhaust the possibilities, there is no counterexample of minimal order to Theorem 1.3, and hence no counterexample at all. Thus Theorem 1.3 holds for all groups of all orders.

We may restate Theorem 1.3 without reference to power graphs as follows.

Corollary 7.7 Let $G$ be a finite group of order $n$. Then $\sum_{d \mid n}(2 d-\phi(d)) \phi(d) \geq$ $\sum_{g \in G}(2 \mathrm{o}(g)-\phi(\mathrm{o}(g)))$, with equality if and only if $G$ is cyclic.

Acknowledgement The second author thanks his advisors Professor Hassan Yousefi Azari and Professor Ali Reza Ashrafi for providing him with this question as a conjecture. We thank Professor I. Martin Isaacs, from the University of Wisconsin-Madison for his valuable comments. We thank Professor Andrei Kelarev from the University of Newcastle (Australia) for discussion on the background and uses of power graphs of groups.

\section{References}

1. Abawajy, J., Kelarev, A., Chowdhury, M.: Power graphs: a survey. Electron. J. Graph Theory Appl. 1(2), 125-147 (2013) 
2. Amiri, H., Jafarian Amiri, S.M., Isaacs, I.M.: Sums of element orders in finite groups. Commun. Algebra 37, 2978-2980 (2009)

3. Apostol, T.M.: Introduction to Analytic Number Theory. Springer, Berlin (1976)

4. Berkovich, Y.: Groups of Prime Power Order vol. 1. Walter de Gruyter GmbH Co, Berlin (2008)

5. Burton, D.M.: Elementary Number Theory, fifth edn. McGraw-Hill, Boston (2002)

6. Cameron, P.J.: The power graph of a finite group, II. J. Group Theory 13, 779-783 (2010)

7. Cameron, P.J., Ghosh, S.: The power graph of a finite group. Discrete Math. 311, 1220-1222 (2011)

8. Chakrabarty, I., Ghosh, S., Sen, M.K.: Undirected power graphs of semigroups. Semigroup Forum 78, 410-426 (2009)

9. Kelarev, A.V., Quinn, S.J.: A combinatorial property and power graphs of groups. In: Contributions to General Algebra, Vienna, 1999, vol. 12, pp. 229-235. Heyn, Klagenfurt (2000)

10. Kelarev, A.V., Quinn, S.J.: Directed graph and combinatorial properties of semigroups. J. Algebra 251, 16-26 (2002)

11. Kelarev, A.V., Quinn, S.J.: A combinatorial property and power graphs of semigroups. Comment. Math. Univ. Carol. 45, 1-7 (2004)

12. Kelarev, A.V., Quinn, S.J., Smolikova, R.: Power graphs and semigroups of matrices. Bull. Aust. Math. Soc. 63, 341-344 (2001)

13. Mirzargar, M., Ashrafi, A.R., Nadjafi-Arani, M.J.: On the power graph of a finite group. Filomat 26(6), 1196-1203 (2012)

14. Rose, J.S.: A Course on Group Theory. Dover, New York (1994) 